\title{
вмJ Global Health Burden of physical, psychological and social ill-health during and after pregnancy among women in India, Pakistan, Kenya and Malawi
}

\author{
Mary McCauley, ${ }^{1}$ Barbara Madaj, ${ }^{1}$ Sarah A White, ${ }^{1}$ Fiona Dickinson, ${ }^{1}$ \\ Sarah Bar-Zev, ${ }^{2}$ Mamuda Aminu, ${ }^{1}$ Pamela Godia, ${ }^{3}$ Pratima Mittal, ${ }^{4}$ Shamsa Zafar, ${ }^{5}$ \\ Nynke van den Broek ${ }^{1}$
}

To cite: McCauley M, Madaj B, White SA, et al. Burden of physical, psychological and social ill-health during and after pregnancy among women in India, Pakistan, Kenya and Malawi. BMJ Glob Health 2018;3:e000625. doi:10.1136/ bmjgh-2017-000625

Handling editor Seye Abimbola

- Additional material is published online only. To view, please visit the journal online (http://dx.doi.org/10.1136/ bmjgh-2017-000625).

Received 30 October 2017 Revised 21 March 2018 Accepted 24 March 2018

Check for updates

${ }^{1}$ Centre for Maternal and Newborn Health, Liverpool School of Tropical Medicine, Liverpool, UK

${ }^{2}$ Centre for Maternal and Newborn Health, Lilongwe Office, Lilongwe, Malawi

${ }^{3}$ Centre for Maternal and Newborn Health, Nairobi Office, Nairobi, Kenya

${ }^{4}$ Department of Obstetrics and Gynaecology, Safdarjung Hospital, New Delhi, India ${ }^{5}$ Child Advocacy International Pakistan, Islamabad, Pakistan

Correspondence to

Dr Mary McCauley;

mary.mccauley@Istmed.ac.uk

\section{ABSTRACT}

Introduction For every woman who dies during pregnancy and childbirth, many more suffer ill-health, the burden of which is highest in low-resource settings. We sought to assess the extent and types of maternal morbidity. Methods Descriptive observational cross-sectional study at primary-level and secondary-level healthcare facilities in India, Pakistan, Kenya and Malawi to assess physical, psychological and social morbidity during and after pregnancy. Sociodemographic factors, education, socioeconomic status (SES), quality of life, satisfaction with health, reported symptoms, clinical examination and laboratory investigations were assessed. Relationships between morbidity and maternal characteristics were investigated using multivariable logistic regression analysis.

Results 11454 women were assessed in India (2099), Malawi (2923), Kenya (3145), and Pakistan (3287). Almost 3 out of 4 women had $\geq 1$ symptoms $(73.5 \%)$, abnormalities on clinical examination (71.3\%) or laboratory investigation (73.5\%). In total, $36 \%$ of women had infectious morbidity of which $9.0 \%$ had an identified infectious disease (HIV, malaria, syphilis, chest infection or tuberculosis) and an additional $32.5 \%$ had signs of early infection. HIV-positive status was highest in Malawi (14.5\%) as was malaria (10.4\%). Overall, $47.9 \%$ of women were anaemic, $11.5 \%$ had other medical or obstetric conditions, $25.1 \%$ reported psychological morbidity and $36.6 \%$ reported social morbidity (domestic violence and/ or substance misuse). Infectious morbidity was highest in Malawi (56.5\%) and Kenya (40.4\%), psychological and social morbidity was highest in Pakistan (47.3\%, 60.2\%). Maternal morbidity was not limited to a core at-risk group; only $1.2 \%$ had all four morbidities. The likelihood of medical or obstetric, psychological or social morbidity decreased with increased education; adjusted OR $(95 \% \mathrm{Cl})$ for each additional level of education ranged from 0.79 (0.75 to 0.83$)$ for psychological morbidity to 0.91 (0.87 to 0.95 ) for infectious morbidity. Each additional level of SES was associated with increased psychological morbidity (OR $1.15(95 \% \mathrm{Cl} 1.10$ to 1.21$))$ and social morbidity (OR 1.05 (95\% Cl 1.01 to 1.10)), but there was no difference regarding medical or obstetric morbidity. However, for each morbidity association was heterogeneous between countries.

Conclusion Women suffer significant ill-health which is still largely unrecognised. Current antenatal and postnatal

\section{Key questions}

What is already known?

- Non-life-threatening maternal morbidity is a new concept internationally.

- This is the first study to comprehensively measure the burden of non-acute maternal morbidity during and after pregnancy using a standardised approach to assess the physical, psychological and social components of ill-health in combination with objective clinical and laboratory measurements.

\section{What are the new findings?}

- Almost three out of four women had $\geq 0$ ne symptoms $(73.5 \%)$, abnormalities on clinical examination $(71.3 \%)$ or laboratory investigation (73.5\%).

- In total, $9.0 \%$ of women had an identified infectious disease (HIV, malaria, syphilis, chest infection or tuberculosis); $32.5 \%$ had signs of early infection, with a presumptive source of infection identifiable in more than two-thirds of women using simple clinical algorithms and point-of-care tests.

- Overall, $47.9 \%$ of women were anaemic, $11.5 \%$ were diagnosed with other medical or obstetric morbidity, $25.1 \%$ of women reported psychological morbidity and $36.6 \%$ reported social morbidity (domestic violence and/or substance misuse).

- Maternal morbidity was not limited to a core 'at-risk' group; only $1.2 \%$ of women had a combination of all four morbidities.

care packages require adaptation if they are to meet the identified health needs of women.

\section{INTRODUCTION}

All women have the right to the highest attainable standard of health and well-being. ${ }^{1}$ Health is a state of complete (physical, psychological and social) well-being and not merely the absence of disease or infirmity. ${ }^{2}$ Commonly, the number of women who die 


\section{Key questions}

\section{What do the new findings imply?}

- This study for the first time highlights a significant burden of ill-health during and after pregnancy that has largely been 'hidden' and/or underestimated.

- At present, when women attend for antenatal and postnatal care in low-income and middle-income settings, their heath needs are not assessed; and point-of-care tests and screening for psychological and social morbidity are not routinely available at primary and secondary level.

- It is important to use the data from this study to improve the content and quality of antenatal and postnatal care packages so that women's health needs during and after pregnancy are assessed and that individualised care which meets the identified health needs of each woman is provided.

during and after pregnancy (maternal mortality) is used as an international health indicator and the new Sustainable Development Goal (3.1) aims to reduce the global maternal mortality ratio to $<70$ per 100000 live births by $2030 .{ }^{3}$ However, the number of women who die only represent the 'tip of the iceberg'. ${ }^{4}$ The shape of this 'iceberg' and the true magnitude of, and relative proportion for, each category including maternal morbidity, is currently poorly documented. For every woman who dies, an estimated 20 or 30 more suffer morbidity related to pregnancy and childbirth. ${ }^{56}$ However, these estimates are not based on standard, well-documented methodology and have limited usefulness for informing strategy. There is also an assumption that there is a relationship and continuum, such that maternal morbidity may increase the risk of more severe acute maternal morbidity (SAMM) and subsequently maternal death. Similarly, prevention or treatment of ill-health is expected to result in fewer women with life-threatening morbidity or death. While the underlying causes of maternal mortality and type of maternal morbidity may not be simply connected, it is recognised that many women suffer both long-term and short-term consequences of pregnancy and childbirth, the major burden of which rests on women living in low-income and middle-income countries (LMIC). ${ }^{7}$

Until now, research has focused on severe and life-threatening acute maternal morbidity, also termed maternal 'near miss', which has now been well defined and generally is assessed at secondary or tertiary care levels. ${ }^{8}$ It is estimated that up to $15 \%$ of women will have a complication during pregnancy, childbirth, or in the postnatal period, which will require emergency obstetric care. ${ }^{9}$ Similarly, the recent Global Burden of Disease Study estimates related to pregnancy are based on modelling for acute complications during pregnancy and childbirth only. ${ }^{10}$ In contrast, morbidity which is not immediately life-threatening and/or morbidity perceived as ill-health by the woman herself, is poorly documented.

A recent suggested definition for non-severe maternal morbidity is 'any health condition attributed to and/or complicating pregnancy, and childbirth that has a negative impact on the woman's well-being. ${ }^{11}$ Currently, there is a lack of understanding of what type and extent of ill-health women suffer during and after pregnancy and to date the burden of non-severe maternal morbidity has not been measured in an approach that is comprehensive, standardised or comparable across different settings.

There are a number of previous studies that have used a variety of clinical or proxy indicators (eg, hospital admission, complications of labour, serious adverse incidents) or documented specific single or multiple known disease conditions only (eg, pre-eclampsia, haemorrhage) over different time frames. ${ }^{812}$ However, in the available literature, sample sizes are limited and the indicators used are not consistent. The Global Burden of Disease Study reported that non-fatal dimensions of disease and injury are more important than ever before and highlighted that currently limited information is available for maternal ill-health. ${ }^{10}$ Similarly, health and well-being are centrally positioned in the 2030 Agenda for sustainable development and there is a call for more accurate measures of and data on health, including for maternal morbidity.

In this study, we therefore sought to measure maternal morbidity in a comprehensive and standardised manner by asking women about symptoms, conducting clinical examination for signs and point-of-care investigations to assess obstetric, medical and infectious morbidity. In addition, psychological as well as social ill-health was assessed using both objective and subjective measures.

Our primary objective was to assess the prevalence and type of maternal morbidity during and after pregnancy in women living in low-income and middle-income settings. As a secondary objective, we explore the relationship between educational level and socioeconomic status (SES) of women and medical or obstetric, infectious, psychological and social morbidity.

\section{METHODS}

\section{Study design and settings}

We conducted a descriptive observational cross-sectional study in India, Pakistan, Kenya and Malawi. In three of these four countries, geographical areas were purposively selected to include both urban and rural women seeking care at primary as well as secondary level healthcare facilities (Pakistan, Islamabad Capital Territory (ICT) and Punjab; Kenya, Central; Malawi, South). In India, the study population was from the largest public health hospital in New Delhi, which provides routine antenatal (ANC) and postnatal care (PNC) to a large and diverse catchment population. In Pakistan, two hospitals in ICT and all three district hospitals for three districts in Punjab, together with five health centres selected using simple random sampling were included. For Kenya and Malawi, in each district, to reflect a cross-section of two levels of healthcare (primary and secondary) the main hospital was purposively selected and in addition two health centres that referred to this hospital were selected 
using simple random sampling. This provided a sample of 12 secondary and 17 primary care level facilities (see online supplementary table 1 ).

With regard to healthcare coverage and uptake, the proportion of women who attend for at least one ANC visit is $75 \%$ in India, $73 \%$ in Pakistan, $92 \%$ in Kenya and $96 \%$ in Malawi. ${ }^{13}$ For skilled attendance at birth (or institutional delivery), this is up to $81 \%$ in India, $61.8 \%$ in Pakistan, $89.8 \%$ in Kenya and $90 \%$ in Malawi. ${ }^{14}$

\section{Participants}

All women attending for ANC, delivery or PNC at the study healthcare facilities were eligible for inclusion. Women with possible SAMM and/or who were too ill to participate (eg, altered conscious level as a result of eclampsia, receiving treatment for an acute complication, admission to high dependency unit) were excluded. Similar numbers of women were recruited at five stages of pregnancy-early antenatal ( $\leq 20$ weeks), late antenatal ( $>21$ weeks), delivery (within 24 hours of birth), early postnatal (days 1-7) and late postnatal (weeks 1-12) (see online supplementary table 2). Women were recruited sequentially between December 2014 and September 2015 until the target sample size for each assessment point was reached in each healthcare facility. All women who consented to take part in the study were interviewed and had a full clinical examination and basic urine and serological investigations performed by trained healthcare providers. Data were collected using a newly developed and standardised structured questionnaire formatted onto iPads in India, Pakistan and Kenya. Paper questionnaires were used in Malawi.

Each woman was only enrolled once in the study and was assessed by a trained healthcare provider (including nurse-midwives and/or other staff cadres who were responsible for providing ANC and PNC in that setting). Training in how to conduct the full assessment (including full history taking, clinical and obstetric examination, point-of-care testing and investigations) was provided by the same team of research leads in each setting and included practical assessment of competency and observed assessments. In each setting, the data collection tool was piloted and checked with regard to consistent use and understanding of the language used. Data collection was supervised by the most senior healthcare provider in each setting. The training included sessions to ensure healthcare providers knew when referral was needed and could provide this. Finally, particular emphasis was placed on professional conduct and the need for confidentiality, privacy and respectful care. Supervisors (senior healthcare providers) were appointed at secondary level and checked (via direct observation) $10 \%$ of all assessments conducted, and, were also available to provide advice regarding women who required referral. Weekly supervisory and coordination meetings (virtual) were arranged with the supervisory team based in the UK for each setting.

\section{Data collection}

Demographics including age, marital status, occupation and educational level (measured as level completed: none, primary, secondary or tertiary) were assessed. SES was derived using Kuppuswamy's scale in India and Pakistan (using women as head of household). ${ }^{15}$ SES was defined using wealth index derived using principal component analysis for Malawi and Kenya. ${ }^{16} 17$

Current physical symptoms were assessed using 76 questions covering six organ systems-cardiopulmonary, gastrointestinal, musculoskeletal, urogynaecology, obstetric and breast, and miscellaneous (dermatology, endocrine, neurological, immunology, ear-nose-throat).

Psychological morbidity was assessed using the Edinburgh Postnatal Depression Scale (EPDS) with depression defined as an EPDS score of $\geq 10 .{ }^{18}$ Questions regarding quality of life (QOL) and satisfaction with health were derived from the WHO 'QOL Spirituality, Religiousness and Personal Beliefs (SRPB)' questionnaire. ${ }^{19}$ The 'Hurt, Insulted, Threatened, Screamed at' (HITS) questionnaire was used to assess domestic violence first from the husband or partner and second from other family members (with a score of $>10$ indicating significant abuse).$^{20}$ Four subquestions from the 'Alcohol, Smoking and Substance Involvement Screening Test' questionnaire were included. ${ }^{21}$

Clinical observations pulse rate (PR), respiratory rate (RR), blood pressure (BP) and oral temperature (T) were measured and the conjunctiva, sclera, breast, abdomen (general and obstetric) were examined. Inspection of the perineum and/or speculum examination was only conducted if clinically indicated (eg, for symptoms of vaginal discharge or bleeding).

\section{Assessment of morbidity}

Occurrence of each symptom and sign was individually evaluated. Where possible, documentation of symptoms, signs and investigations were 'triangulated' and grouped as indicative of specific maternal morbidities, for example, headache, visual disturbances, upper abdominal pain, raised BP plus proteinuria indicative of pre-eclampsia. A reported symptom of a productive cough of $>2$ weeks was used to indicate either a possible chest infection or suspected tuberculosis (TB). Antenatal haemorrhage was defined as women who reported bleeding per vagina during pregnancy and/or who had this confirmed on examination. Incontinence was defined as women who reported any leakage of urine and/or had this confirmed on examination.

Urinalysis was performed using Multistix GP. A simple finger prick test was used to obtain one capillary $(<0.5 \mathrm{~mL})$ of blood for use in four rapid diagnostic point-of-care tests: haemoglobin (HemoCue), malaria (Humasis), syphilis and HIV (SD BIOLINE HIV/Syphilis Duo) and $\mathrm{C}$ reactive protein (CRP) (QuickRead). CRP could be measured in all settings except in some healthcare facilities at primary level in Malawi and Pakistan. Anaemia was classified as haemoglobin $<110 \mathrm{~g} / \mathrm{L} .{ }^{22}$ Hypertension was 
classified as $\mathrm{BP} \geq 140 / 90 .{ }^{23}$ Pre-eclampsia was defined as $\mathrm{BP} \geq 140 / 90$, and proteinuria ( $\mathrm{PR} \geq++$ on urinalysis) after 20 weeks gestation. ${ }^{23}$

We amended the systematic inflammatory response syndrome (SIRS) score ${ }^{23}$ to define possible early infection as the presence of two or more of the following: (1) $\mathrm{T}>38^{\circ} \mathrm{C}$ or $<36^{\circ} \mathrm{C}$, (2) $\mathrm{PR}>90$ beats per min; (3) $\mathrm{RR}>20$ breaths per min or (4) raised CRP (defined as $>5 \mathrm{mg}$ / $\mathrm{dL}$ at each assessment point, apart from after delivery (within 24 hours of birth) where raised CRP was defined as $>10 \mathrm{mg} / \mathrm{dL}$ ).

Summative physical morbidity was categorised as (1) infectious or (2) medical or obstetric. Infectious morbidity included: HIV, malaria, syphilis, chest infection or suspected TB, and a SIRS score of $\geq 2$. Medical or obstetric morbidity included: anaemia, hypertension, pre-eclampsia, antenatal haemorrhage and incontinence. We defined psychological morbidity as an EPDS score of $\geq 10$ and/or thoughts of self-harm. ${ }^{18}$ We defined social morbidity as a woman reporting any domestic violence (HITS score $>4$ ) and/or any substance misuse.

\section{Sample size calculation and statistical analysis}

In Pakistan, Kenya and Malawi for each of the five assessment points, data were collected for a minimum of 576 women across two levels of healthcare facility (primary and secondary) selected by stratified cluster sampling. In India, as the study was conducted in one facility (secondary level) a cluster sampling approach was not required, giving an amended sample size of 1920 with a minimum of 384 women per assessment point. This sample size had $95 \%$ power to detect the presence of any morbidity with a prevalence $>1 \%$. With a sample size exceeding 2000, the margins of error for estimation of prevalence of $1 \%, 10 \%$ and $50 \%$ do not exceed $0.5 \%$, $1.4 \%$ and $2.2 \%$, respectively. Data analysis was performed using SPSS V.22 and Stata V.12.1. Descriptive statistics are provided for relevant characteristics by country; these provide estimates of the prevalence of these characteristics. For each morbidity, multivariable logistic regression analyses were performed using SES and education category as covariates. This assumes a common OR for the impact of a change to the next category for a covariate. Data for all four countries were combined in one set of analyses as well as data being analysed separately for each country. Analyses of data for all four countries included interactions between country and each of SES and education to examine evidence of heterogeneity among countries. Likelihood ratio tests were used to compare logistic regression models and derive corresponding $\mathrm{p}$ values. A $p$ value of $<0.05$ was used to determine statistical significance and estimates provided with robust $95 \%$ CIs. OR were estimated using multivariable logistic regression models for each morbidity. Since there is considerable association between SES and education category, the multivariable models are reported to enable the contribution of each, after accounting for the other, to be distinguished. Unless otherwise stated, all percentages reported use the total sample size for the relevant country. Where a substantial proportion of women $(>10 \%)$ had data missing for a variable this is reported in the tables. Absence of a response for a question used in derivation of morbidity was treated as absence of the sign/symptom/ abuse or the most positive response for depression.

\section{Ethics}

Written informed consent was obtained from each woman who participated in the study.

\section{RESULTS \\ Study population}

A total of 11454 women across four LMICs were assessed: India (2099), Malawi (2923), Kenya (3145) and Pakistan (3287) with similar numbers of women assessed at each of the five stages of pregnancy (see online supplementary table 2). The refusal rate was low (between $1.1 \%$ and $2.5 \%$ ) in each country and was mainly because of lack of time for the woman to participate. Following recruitment and after taking a clinical history, 150 women (1.3\%) declined clinical examination and 138 women (1.2\%) declined laboratory investigations.

\section{Quality of life and satisfaction with health}

For all countries combined, the majority of women reported having a good QOL $(75.9 \%)$ and being satisfied with their health $(78.2 \%)$. This was least well scored in Pakistan $(56.4 \%$ satisfied with QOL and $57.4 \%$ with health) and highest in Malawi (95\% satisfied with QOL and $95.5 \%$ with health).

\section{Symptoms}

A detailed clinical history was obtained. Almost threequarter of all women $(8425 ; 73.5 \%)$ reported at least one clinical symptom with a median (IQR) of $4.2(0-27)$ symptoms per woman (figure 1). Women in Pakistan and India most frequently reported symptoms $(92.1 \%$ and $90.4 \%$ of women, respectively). Overall, when categorised by organ system, symptoms were most frequently related to the gastrointestinal tract $(23.9 \%$ of all symptoms reported) followed by obstetric and breast (16.7\%), urogynaecological $(16.1 \%)$ cardiopulmonary $(15.5 \%)$, musculoskeletal $(12.8 \%)$ and miscellaneous (including immunology, dermatology and endocrine) (15.0\%). There were slight variations in the trend but the most common was gastrointestinal symptoms in all four countries followed by cardiopulmonary symptoms in Malawi and Kenya, urogynaecological in Pakistan and obstetric or breast related in India.

\section{Psychological and social morbidity}

Psychological and social morbidity were assessed as part of the clinical history. Psychological morbidity was noted in one in four women $(25.1 \%$ ), with $22.2 \%$ having an EPDS $\geq 10 \%$ and $15.2 \%$ of women reporting thoughts of self-harm (table 1). Psychological morbidity was highest in Pakistan $(29.8 \%)$ and lowest in Kenya (4.5\%). 


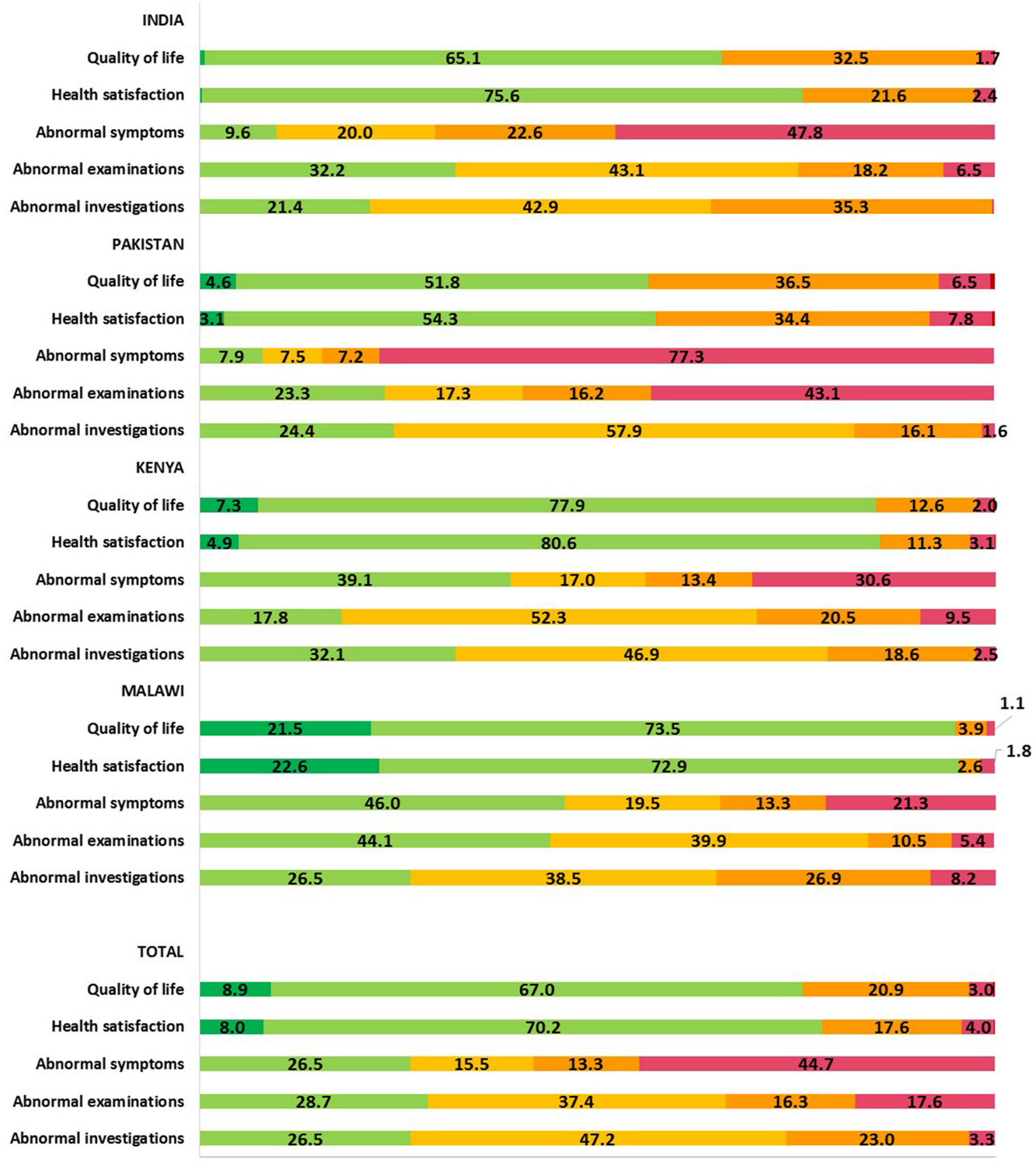

\begin{tabular}{|l|l|l|c|c|c|}
\hline & $\begin{array}{c}\text { Satisfaction } \\
\text { with health }\end{array}$ & Quality of life & $\begin{array}{c}\text { Number of } \\
\text { symptoms }\end{array}$ & $\begin{array}{c}\text { Number of } \\
\text { abnormal } \\
\text { examinations }\end{array}$ & $\begin{array}{c}\text { Number of } \\
\text { abnormal } \\
\text { investigations }\end{array}$ \\
\hline & Very satisfied & Very good & - & - & - \\
\hline & Satisfied & Good & 0 & 0 & 0 \\
\hline & $\begin{array}{l}\text { Neither satisfied } \\
\text { nor dissatisfied }\end{array}$ & $\begin{array}{l}\text { Neither good nor } \\
\text { poor }\end{array}$ & 1 & 1 & 1 \\
\hline & Dissatisfied & Poor & 2 & 2 & 2 \\
\hline & Very dissatisfied & Very poor & $\geq 3$ & $\geq 3$ & $\geq 3$ \\
\hline
\end{tabular}

Figure 1 Histogram of quality of life, satisfaction with health, number of symptoms, number of abnormal clinical examinations and number of abnormal laboratory investigations (percentage of women assessed by country and for all countries combined).

Overall, 3887 women $(33.9 \%)$ reported domestic violence; more frequently from the husband $(26.3 \%)$ than from another family member (15.8\%) (table 1 ). Overall, $8.9 \%$ of women suffered significant domestic violence (HITS score $>10$ ) with larger proportions of women in Pakistan reporting both any, and, significant domestic violence. Use of alcohol, sedatives or inhalants was not common with
$6.5 \%$ of women reporting using any of these substances within the past 3 months and $1.7 \%$ of women requiring intervention for substance misuse (table 1).

Infectious, medical and obstetric morbidity

In total, $73.1 \%$ of women had at least one or more abnormal finding on clinical examination (figure 1). 


\begin{tabular}{|c|c|c|c|c|c|c|}
\hline \multicolumn{2}{|l|}{ Category } & India & Pakistan & Kenya & Malawi & Total \\
\hline \multicolumn{2}{|l|}{ Number of women } & 2099 & 3287 & 3145 & 2923 & 11454 \\
\hline \multicolumn{7}{|l|}{ Psychological morbidity (\%) } \\
\hline \multicolumn{2}{|l|}{$E P D S \geq 10$} & 19.3 & 41.6 & 12.3 & 15.6 & 22.9 \\
\hline \multicolumn{2}{|l|}{ Thoughts of self-harm } & 15.6 & 29.8 & 4.5 & 10.1 & 15.2 \\
\hline \multicolumn{2}{|c|}{ EPDS $\geq 10$ and/or thoughts of self-harm } & 19.8 & 47.3 & 13.5 & 16.4 & 25.1 \\
\hline \multicolumn{7}{|l|}{ Social morbidity (\%) } \\
\hline \multicolumn{7}{|l|}{ Domestic violence } \\
\hline \multirow{3}{*}{$\begin{array}{l}\text { Any domestic violence } \\
\text { HITS score }>4\end{array}$} & Husband and/or family & 39.7 & 56.0 & 21.7 & 18.2 & 33.9 \\
\hline & Husband & 38.6 & 37.4 & 18.3 & 13.6 & 26.3 \\
\hline & Family & 10.1 & 31.8 & 6.0 & 12.5 & 15.8 \\
\hline \multirow{3}{*}{$\begin{array}{l}\text { Significant } \\
\text { domestic violence } \\
\text { HITS score>10 }\end{array}$} & Husband and/or family & 6.1 & 21.7 & 3.2 & 4.7 & 8.9 \\
\hline & Husband & 5.1 & 12.5 & 2.4 & 3.0 & 6.0 \\
\hline & Family & 2.7 & 7.5 & 0.5 & 2.6 & 3.4 \\
\hline \multicolumn{7}{|l|}{ Substance misuse } \\
\hline \multicolumn{2}{|c|}{$\begin{array}{l}\text { Use of alcohol, sedatives, inhalants, tobacco in last } \\
3 \text { months }\end{array}$} & 2.7 & 0.2 & 2.0 & 1.7 & 6.5 \\
\hline \multicolumn{2}{|c|}{ Intervention required (ASSIST score>4) } & 0.8 & 1.5 & 1.4 & 2.8 & 1.7 \\
\hline \multicolumn{2}{|c|}{ Domestic violence and/or substance misuse } & 40.3 & 60.2 & 25.0 & 19.8 & 36.6 \\
\hline
\end{tabular}

ASSIST, Alcohol, Smoking and Substance Involvement Screening Test; EPDS, Edinburgh Postnatal Depression Scale; HITS, Hurt, Insulted, Threatened, Screamed at.

The most common findings were: conjunctival pallor $(23.0 \%)$, breast problems $(16.0 \%)$, gum and oral cavity problems $(12.1 \%)$ and abdominal tenderness $(10.9 \%)$ (table 2).

Vaginal examination was only offered, and consent obtained, if clinically indicated; perineum examination was indicated and performed in $54.9 \%$ (6288) of women and speculum examination in $22.3 \%$ (2555) of women; $25.9 \%$ of women had perineal problems (vaginal tears, excoriation, swelling) and $3.3 \%$ were noted to have leakage of urine (table 2). This is equivalent to an overall estimated prevalence of $15.5 \%$ for perineal morbidity across the four countries, but noted to be particularly high among women in Pakistan. Similarly, 36.4\% of women examined by speculum were noted to have abnormal vaginal discharge, and vaginal bleeding was confirmed in $14.8 \%$ giving an overall estimated prevalence of between $2.4 \%$ and $5.1 \%$ in India, Kenya and Malawi, but up to $30.8 \%$ among women in Pakistan (table 2).

Reported symptoms, results of clinical examination and laboratory investigation were combined where appropriate to determine (1) infectious morbidity and (2) medical or obstetric morbidity (table 3 ).

Using an amended SIRS score, $32.5 \%$ of women in whom CRP was measured had early signs of possible infection. In almost two-thirds of cases, a presumptive cause or source of infection could be identified (based on symptoms and clinical examination), which included: gastroenteritis (18.8\%), lower respiratory tract infection
$(13.0 \%)$, sexually transmitted infection $(10.1 \%)$, urinary tract infection $(9.6 \%)$, mastitis $(7.4 \%)$, upper respiratory tract infection $(4.4 \%)$, endometritis $(2.0 \%)$, chorioamnionitis $(1.6 \%)$ and wound infection $(0.6 \%)$.

\section{Comorbidity}

Overall, one or more type of infectious morbidity was identified in $28.0 \%$ of women, and, one or more type of medical or obstetric morbidity in 50.0\% (see online supplementary figure 1 ). Only $22.9 \%$ of all women had no identifiable morbidity and only $1.2 \%$ had all four types of morbidity.

\section{Association between maternal morbidity, education and socioeconomic status}

Separate multivariable analyses for each form of morbidity, which assume a consistent effect of SES and education across country and account for country and SES, found that as education level increases, the likelihood of each morbidity (psychological, social, medical or obstetric) except infectious conditions decreases (table 4). For each additional level of education (primary, secondary or higher) completed, the adjusted odds of morbidity were estimated to reduce by a factor between $0.79(95 \%$ CI 0.75 to 0.83 ) for psychological morbidity and $0.91(95 \%$ CI 0.87 to 0.95 ) for infectious morbidity. Conversely, in these analyses, as SES increases (for a fixed education category), the likelihood of morbidity increases for psychological (OR 1.15 (95\% CI 1.10 to 1.21)) and 
Table 2 Abnormal findings on clinical examination by country and for all countries combined

\begin{tabular}{|c|c|c|c|c|c|c|}
\hline Country & & India & Pakistan & Kenya & Malawi & Total \\
\hline Number of women assessed* & & 2099 & 3287 & 3145 & 2923 & 11454 \\
\hline \multicolumn{7}{|l|}{ Clinical examination-general } \\
\hline Variable & Definition & $\%$ & $\%$ & $\%$ & $\%$ & $\%$ \\
\hline $\begin{array}{l}\text { Pulse rate } \\
\text { (PR, beats per min) }\end{array}$ & $\mathrm{PR} \leq 50$ or $\mathrm{PR} \geq 100$ & 0.7 & 1.8 & 7.3 & 7.4 & 4.5 \\
\hline $\begin{array}{l}\text { Respiratory rate (RR, breaths per } \\
\text { min) }\end{array}$ & $\mathrm{RR} \leq 8$ or $\mathrm{RR} \geq 20$ & 0.1 & 39.0 & 72.7 & 43.2 & 42.3 \\
\hline Temperature $\left(\mathrm{T},{ }^{\circ} \mathrm{C}\right)$ & $\mathrm{T} \leq 35$ or $\mathrm{T} \geq 38.0$ & 15.2 & 10.6 & 1.2 & 3.4 & 7.0 \\
\hline Systolic blood pressure (mm Hg) & $\leq 90$ or $\geq 140$ & 2.5 & 7.3 & 7.6 & 4.4 & 5.8 \\
\hline Diastolic blood pressure (mm Hg) & $\leq 45$ or $\geq 90$ & 3.0 & 11.6 & 4.3 & 4.4 & 6.2 \\
\hline Nutritional status (kg/m2) & $\begin{array}{l}\text { Body mass index } \leq 18.5 \mathrm{~kg} / \\
\mathrm{m}^{2}\end{array}$ & 3.0 & 2.5 & 1.5 & 3.0 & 2.5 \\
\hline
\end{tabular}

Clinical examination-by organ system

\begin{tabular}{|c|c|c|c|c|c|c|}
\hline Organ system & Abnormal finding & $\%$ & $\%$ & $\%$ & $\%$ & $\%$ \\
\hline \multirow[t]{6}{*}{ General } & Conjunctival pallor & 40.9 & 43.9 & 6.1 & 4.7 & 23.0 \\
\hline & Sclera & 0.7 & 3.5 & 0.1 & 0.0 & 1.2 \\
\hline & Goitre & 0.2 & 6.3 & 0.2 & 0.2 & 2.0 \\
\hline & Peripheral pitting oedema & 2.8 & 13.9 & 2.6 & 0.9 & 5.4 \\
\hline & Central pitting oedema & 0.3 & 5.7 & 0.3 & 0.5 & 1.9 \\
\hline & Total & 45.0 & 73.3 & 9.3 & 6.3 & 33.5 \\
\hline \multirow[t]{4}{*}{ Skin } & Skin rashes & 3.5 & 9.2 & 1.4 & 0.7 & 3.7 \\
\hline & Skin ulcers & 0.2 & 2.4 & 0.2 & 0.0 & 0.8 \\
\hline & Skin lump or growth & 0.4 & 5.7 & 0.8 & 0.2 & 2.0 \\
\hline & Total & 4.1 & 17.3 & 2.4 & 0.9 & 6.5 \\
\hline \multirow[t]{4}{*}{ Oral cavity } & Bleeding gums & 0.8 & 14.5 & 5.3 & 0.1 & 6.0 \\
\hline & Oral thrush & 0.3 & 9.3 & 0.5 & 0.3 & 3.0 \\
\hline & Mouth ulcers & 1.6 & 9.3 & 0.3 & 0.1 & 3.1 \\
\hline & Total & 2.7 & 33.1 & 6.1 & 0.5 & 12.1 \\
\hline \multirow[t]{6}{*}{ Breast } & Cracked nipples & 0.3 & 12.3 & 1.3 & 0.8 & 4.2 \\
\hline & Abnormal engorgement & 3.3 & 20.6 & 0.7 & 0.5 & 6.8 \\
\hline & Abnormal tenderness & 1.3 & 11.1 & 1.0 & 0.8 & 3.9 \\
\hline & Abscess & 0.3 & 0.9 & 0.2 & 0.3 & 0.4 \\
\hline & Lump & 1.2 & 0.8 & 0.6 & 0.3 & 0.7 \\
\hline & Total & 6.4 & 45.7 & 3.8 & 2.7 & 16.0 \\
\hline \multirow[t]{3}{*}{ Abdominal } & Abnormal tenderness & 25.7 & 17.9 & 2.4 & 1.3 & 10.9 \\
\hline & Abnormal mass & 0.1 & 6.3 & 0.2 & 0.0 & 1.9 \\
\hline & Total & 25.8 & 24.2 & 2.6 & 1.3 & 12.8 \\
\hline \multicolumn{7}{|c|}{ Clinical examination - perineum and speculum examination $\dagger$} \\
\hline \multicolumn{2}{|c|}{ Number of women with indication and assessed } & 563 & 2654 & 2225 & 846 & 6288 \\
\hline \multicolumn{2}{|l|}{ Perineum } & $\%$ & $\%$ & $\%$ & $\%$ & $\%$ \\
\hline \multicolumn{2}{|c|}{ Leakage of urine } & 0.0 & 7.1 & 0.6 & 0.5 & 3.3 \\
\hline \multicolumn{2}{|l|}{ Excoriation } & 0.0 & 12.0 & 0.5 & 2.2 & 5.5 \\
\hline \multicolumn{2}{|l|}{ Swelling } & 1.2 & 18.2 & 1.1 & 3.2 & 8.7 \\
\hline \multicolumn{2}{|l|}{ Tear } & 0.7 & 18.0 & 5.9 & 7.0 & 10.7 \\
\hline \multicolumn{2}{|l|}{ Subtotal } & 1.9 & 55.3 & 8.1 & 12.9 & 28.2 \\
\hline \multicolumn{2}{|c|}{ Percentage of study sample } & 0.5 & 44.6 & 5.7 & 3.7 & 15.5 \\
\hline \multicolumn{2}{|c|}{ Number of women with indication and assessed } & 365 & 1729 & 316 & 154 & 2555 \\
\hline
\end{tabular}




\begin{tabular}{|c|c|c|c|c|c|}
\hline Country & India & Pakistan & Kenya & Malawi & Total \\
\hline Speculum examination & $\%$ & $\%$ & $\%$ & $\%$ & \\
\hline Abnormal vaginal discharge & 11.8 & 43.5 & 17.2 & 52.6 & 36.4 \\
\hline Abnormal bleeding & 2.2 & 15.1 & 33.4 & 1.3 & 14.8 \\
\hline Subtotal & 14.0 & 58.6 & 50.6 & 53.9 & 51.2 \\
\hline Percentage of study sample & 2.4 & 30.8 & 5.1 & 2.8 & 11.4 \\
\hline
\end{tabular}

*Percentage with abnormal findings calculated as percentage of those who consented to the examination. Percentage of total $(\mathrm{n}=11$ 454) who provided consent for general examination $98.7 \%$, skin and oral cavity examination $92.0 \%$, breast examination $90.0 \%$ and examination of the abdomen $97.4 \%$.

†Vaginal examination was only offered and consent obtained if clinically indicated; for perineum examination $54.9 \%$ of women; for speculum examination $20.1 \%$ of women.

social morbidity (OR 1.05 (95\% CI 1.01 to 1.10$)$ ), but not for medical or obstetric morbidity. For each of the four morbidities, there was however evidence $(\mathrm{p}<0.001$ in all cases) of heterogeneity between the countries in the effects of SES and education on the risk of morbidity (table 4).

For India, the likelihood of psychological morbidity increased with education, with an estimated OR of 1.53

Table 3 Infectious and medical or obstetric morbidity identified per country and for all countries combined (number of women assessed $n=11454$ )

\begin{tabular}{|c|c|c|c|c|c|c|}
\hline \multicolumn{2}{|l|}{ Country } & India & Pakistan & Kenya & Malawi & Total \\
\hline \multicolumn{2}{|l|}{ Number of women* } & 2099 & 3287 & 3145 & 2923 & 11454 \\
\hline \multicolumn{7}{|l|}{ Infectious morbidity (\%) } \\
\hline Condition* & Definition & & & & & \\
\hline HIV & Positive & 0.3 & 0.3 & 3.6 & 14.3 & 4.8 \\
\hline Malaria & Positive & 0.1 & 0.0 & 0.2 & 10.2 & 2.7 \\
\hline Syphilis & Positive & 0.0 & 0.0 & 0.3 & 3.4 & 0.9 \\
\hline $\begin{array}{l}\text { Positive screening for chest } \\
\text { infection/possible TB }\end{array}$ & Symptomatic cough $>2$ weeks & 0.4 & 0.8 & 0.7 & 0.7 & 0.6 \\
\hline \multicolumn{2}{|c|}{ Number of women with CRP measured $\dagger$} & 1873 & 792 & 2984 & 1544 & 7193 \\
\hline $\begin{array}{l}\text { Septic inflammatory response } \\
\text { syndrome† }\end{array}$ & $\begin{array}{l}\text { Any two of the following: } \\
-\mathrm{PR}>90 \text { beats per min } \\
-\mathrm{RR}>20 \text { breaths per min } \\
-\mathrm{T}<36^{\circ} \mathrm{C} \text { or } \mathrm{T}>38^{\circ} \mathrm{C} \\
\text {-Raised } \mathrm{CRP} \mathrm{mg} / \mathrm{dL}\end{array}$ & 15.4 & 25.8 & 38.2 & 45.6 & 32.5 \\
\hline \multicolumn{2}{|l|}{ At least one infectious condition } & 15.9 & 27.0 & 40.4 & 56.5 & 36.0 \\
\hline \multicolumn{7}{|l|}{ Medical or obstetric morbidity (\%) } \\
\hline \multicolumn{2}{|l|}{ Condition } & & & & & \\
\hline Anaemia & $\mathrm{Hb}<110 \mathrm{~g} / \mathrm{L}$ & 61.2 & 68.7 & 23.9 & 41.0 & 47.9 \\
\hline Hypertension & $\mathrm{BP} \geq 140 / 90$, no proteinuria & 1.5 & 4.8 & 1.8 & 0.8 & 2.4 \\
\hline Pre-eclampsia & $\mathrm{BP} \geq 140 / 90$, proteinuria $(\mathrm{PR} \geq++)$ & 0.1 & 1.5 & 0.4 & 0.3 & 0.6 \\
\hline Urine incontinence & $\begin{array}{l}\text { Symptom and/or finding on } \\
\text { examination }\end{array}$ & 1.7 & 9.6 & 1.6 & 1.5 & 3.6 \\
\hline Antenatal haemorrhage & $\begin{array}{l}\text { Symptom and/or finding on } \\
\text { examination }\end{array}$ & 5.3 & 8.2 & 5.2 & 0.6 & 4.9 \\
\hline At least one medical or obstetric & ondition & 62.8 & 71.2 & 27.1 & 41.5 & 50.0 \\
\hline
\end{tabular}

*Where data were missing for a condition, the condition was regarded as being absent. For purposes of deriving morbidities, \% missing was: HIV 9.7\%, malaria 5.3\%, syphilis $8.9 \%$, screening for chest infection/TB 2.0\%, anaemia $1.9 \%$, BP $2.3 \%$, urine incontinence $0.5 \%$.

TCRP was not measured at some primary level facilities in Malawi and Pakistan. Only participants for whom a CRP result was obtained are included in these statistics.

$\mathrm{BP}$, blood pressure; CRP, $\mathrm{C}$ reactive protein; Hb, haemoglobin; PR, pulse rate; RR, respiratory rate; TB, tuberculosis. 


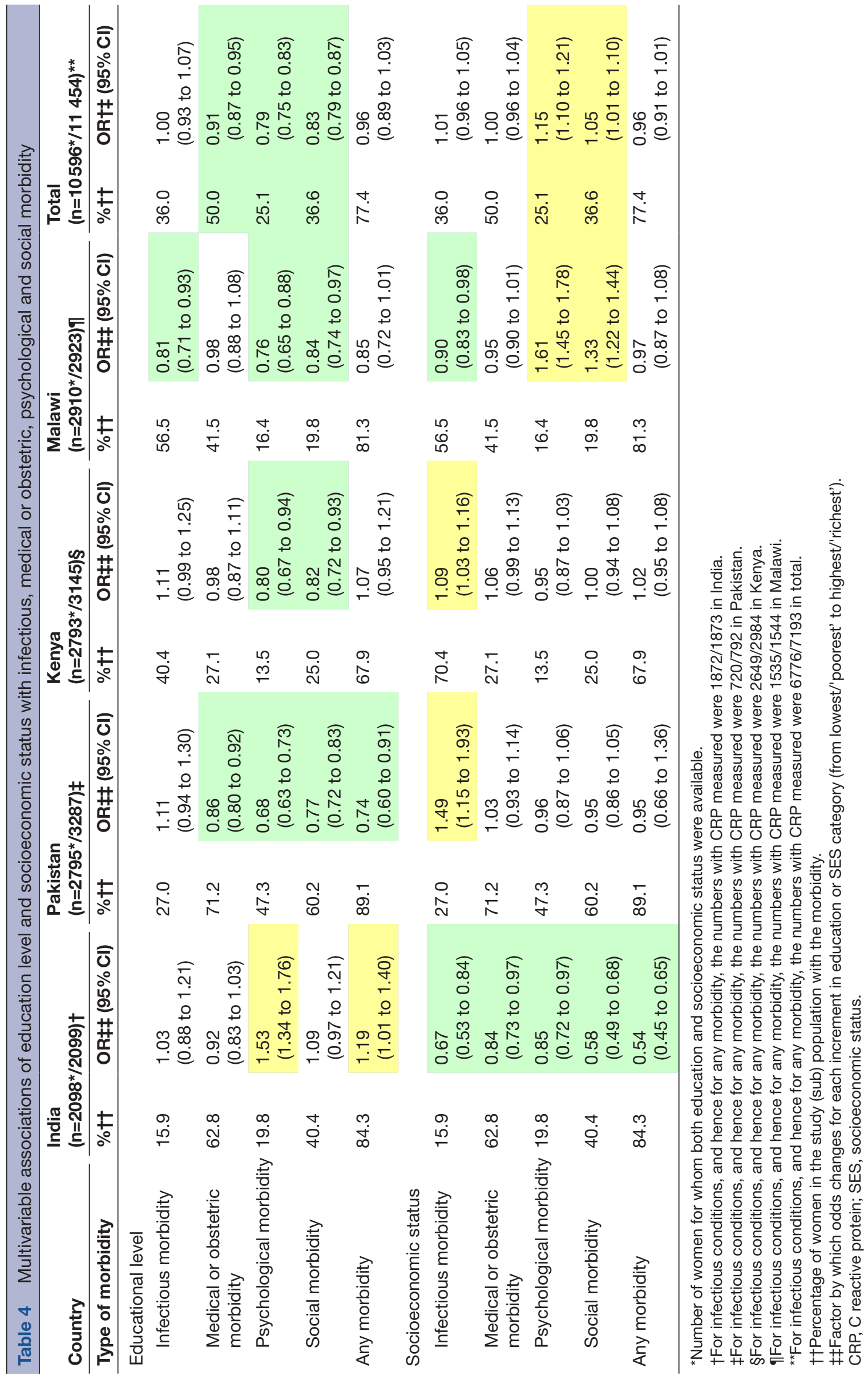


for each additional level of education, and the estimated odds of each morbidity decreased by a factor between 0.58 and 0.85 for each additional level of SES. For Pakistan, the estimated odds associated with education were similar to those for all countries; however, for infectious morbidity the estimated odds increased with each additional level of SES by a factor of 1.49 and no association with SES was detected for other morbidities. For Kenya, the estimated odds associated with education for non-physical morbidities were similar to those for all countries, with no association with education level detected for either of the physical morbidities. However, for infectious morbidity the estimated odds increase with each additional level of SES by a factor of 1.09 and no association with SES was detected for other morbidities. For Malawi, the estimated odds associated with education for non-physical morbidities were similar to those for all countries; a similar association (OR 0.81) with education level was also detected for infectious morbidity. For infectious morbidity, the estimated odds also decreased with each additional level of SES by a factor of 0.90. For non-physical morbidities, the odds of morbidity were estimated to increase more substantially with each additional level of SES than in analyses for other countries.

\section{DISCUSSION}

\section{Statement of principal findings}

Despite most women reporting good QOL (75.9\%) and satisfaction with health $(78.2 \%)$, almost three out of four women reported symptoms $(73.5 \%)$, had abnormal findings on clinical examination $(71.3 \%)$ or on simple laboratory investigation $(73.5 \%)$. Overall, one in four women (25.1\%) had symptoms of psychological morbidity using the EPDS as a screening tool. More than one in three women $(36.6 \%)$ reported social morbidity (domestic violence and/or substance misuse), with $15.6 \%$ of women demonstrating both psychological and social morbidity.

Most women $(8834 ; 77.1 \%$ ) had at least one type of identifiable morbidity and a very small number of women $(138 ; 1.2 \%)$ suffer all four types of morbidity, which suggests that morbidity is not limited to a core 'at-risk' group of women. Women in Pakistan tended to report more physical, psychological and social morbidity. Of all women tested, $47.4 \%$ of women were anaemic with the highest prevalence among women from India and Pakistan. Using our amended SIRS score, $35.5 \%$ of women had clinical signs of early infection. When simple clinical algorithms were applied, a source of infection could be identified in more than $65 \%$ of cases most frequently presumed to be gastroenteritis, lower respiratory tract infection or a sexually transmitted infection. The prevalence of HIV, malaria and syphilis (as confirmed by point-of-care testing) was below $5 \%$ in all settings except Malawi.

Combining all countries and accounting for SES, as education level increases the likelihood of medical or obstetric, psychological and social morbidity decreases.
Conversely, after accounting for education level, as SES increases, the likelihood of psychological and social morbidity increases. However, these effects are not consistent across the four countries, with the direction of impact (if any) of education or SES changing for some morbidities. SES was measured in differing ways between the two continents studied. This may account in part for some differences, however the estimated ORs for SES were statistically significantly in differing directions for infectious conditions in both continents. There are important differences among the four countries (such as higher prevalence of HIV and malaria in Malawi and higher prevalence of psychological and social morbidity, and morbidity in general, except for infectious morbidity in Pakistan). These findings require further research.

\section{Strengths of the study}

To the best of our knowledge, this is the first study to assess maternal morbidity during and after pregnancy using a new integrated comprehensive tool to assess self-reported subjective physical, psychological and social components of ill-health in combination with objective clinical and laboratory measurements performed by trained healthcare providers. The low refusal rate suggests that women attending for care at primary and secondary level healthcare facilities in LMIC settings welcome such an assessment of their health. Our study also shows that it is feasible and acceptable for healthcare providers in these settings to screen women for different types of ill-health during routine healthcare consultations using validated questions and point-of-care diagnostic investigations that can be applied in low-resource settings. This study, for the first time, provides standardised baseline measurements of maternal morbidity that have been calculated using clear and concise methodology, enabling comparisons between different settings and countries. Further strengths of this study are that both subjective (self-reported symptoms) and objective measures (examination and investigations) are included thus helping document which areas of ill-health are considered important by women themselves, and, does so for up to 12 weeks after childbirth.

This study presents estimates of non-severe maternal morbidity. It is important to recognise that this does not include estimates of incidence of SAMM and/or maternal 'near miss', which are separately defined, and, for which global estimates are available. The sum total of both severe or acute maternal morbidity and maternal morbidity as defined and assessed in this study, clearly constitute a very significant burden of disease and risk for women at the time of pregnancy and childbirth. Much of this burden of disease could be diagnosed, treated and prevented, if, the current ANC and PNC care packages are expanded with regard to both content and quality.

\section{Weaknesses of the study}

This was an observational cross-sectional study using purposive sampling that represents convenience samples 
as opposed to population samples from a representative framework and hence is limited in terms of the sections of the population it represents. SES is a complex factor that needs to be carefully considered. The results for the study population in India seem to be contrary to other countries with higher education level being associated with increased risk of psychological morbidity while higher SES associated with reduced risk of morbidity. At present, there is no international consensus on how to measure SES. This requires further research. Our findings are however in line with those reported in the Global Burden of Disease studies. These studies included a new composite indicator Socio demographic Index (SDI), which includes measures of per capita income, education and fertility rate. Country-specific drivers of disease burden where found to be higher than expected based on SDI. ${ }^{24}$

This study population includes women who accessed a healthcare facility for routine ANC, delivery or PNC and excludes those women who did not access care. Thus, the prevalence of morbidity can be expected to be higher (for women who are ill but not able to access care) or lower (for women who feel well and do not see the need to access care). However, this was primarily a pragmatic study to assess the needs of women who access routine care during or after pregnancy and childbirth. At least $77 \%$ of women access ANC once in sub-Saharan Africa and Southeast Asia. ${ }^{13}$ ANC and PNC care packages were designed several decades ago and are currently in process of being updated. It is important that these care packages are expanded to meet the identified health needs of women.

Absence of a response for any morbidity was taken as absence of the sign/symptom/abuse/contribution to depression score. For most instances of isolated missing values, this approach seems likely to be correct. However, lack of recording of presences would result in underestimation of the prevalence of the morbidity. The amount of data missing would not result in $>5 \%$ relative change in estimate. For the analyses of associations such errors of classification would also reduce the power to detect association.

\section{Strengths and weaknesses in relation to other studies, discussing particularly any differences in results}

As this is the first study to assess physical, psychological and social components of ill-health comprehensively in four LMIC, there is little data in the literature against which to compare the overall burden of maternal morbidity. There are early reports of population surveys or health camps, which tried to identify maternal ill-health in Uganda and Egypt. ${ }^{12} 25$ The community-based survey in Egypt reported up to $82.8 \%$ of women having morbidity before, during or after pregnancy, respectively. One prospective study followed up 280 women in rural India for 5 years and reported pregnancy-related morbidity in $30 \%$ of women. ${ }^{5}$ A larger cross-sectional community-based study from India reported that among 3600 women, $41 \%$ and
$42.9 \%$ had morbidity during and after the last pregnancy, respectively. ${ }^{26}$ In a more recent study, $50.0 \%$ of 1732 women in Malawi and 53.0\% of 1727 women in Pakistan had a least one medical or obstetric morbidity (infective or non-infective) but this relied only on solicited symptoms and limited clinical examination. ${ }^{7}$ Almost none of the previous studies included social and psychological aspects of ill-health and none included simple one-stop laboratory investigations which are now available.

Criteria for reported physical morbidity (symptoms and signs) can be subjective and this is the first study to objectively demonstrate that it is feasible and acceptable to screen women comprehensively for all components of health (physical, psychological and social) during a 'routine' health facility visit. There is clearly a need to expand the current assessment of health beyond taking a simple history of physical symptoms related to the pregnancy. Rapid point-of-care tests for the main global infections such as HIV, syphilis and malaria and for anaemia have been developed but are rarely available and/or used for women who access ANC and/or PNC. Until now, there has been no point-of-care test available to screen for TB during and/or after pregnancy. The standard screening has consisted of asking whether a woman has a history of a persistent productive cough after which sputum testing is organised. Unless more regular and accurate screening is introduced, it is very likely that, as in this study, this results in an underestimation of the prevalence of TB during and after pregnancy. This is likely to be especially so in Asia where screening for TB is not linked to (optout) HIV screening, which is however in place across many sub-Saharan African countries.

The EPDS was used to measure psychological morbidity. The EPDS is easy to administer, has proven to be an effective screening tool and has been previously been validated in 12 countries in 14 languages. ${ }^{27}$ The questionnaire is designed to identify possible symptoms of depression and anxiety and is not a diagnostic tool per se. The stage of pregnancy (antenatal or postnatal) may contribute to the variation in the prevalence of psychological morbidity. Based on three systematic reviews, these figures are estimated as $4.3 \%-25 \%$ of women during pregnancy; and $3.2 \%-48.0 \%$ of women following childbirth in LMIC. ${ }^{28-30}$ These results are in line with the findings of this study that depression was a significant problem along the continuum of pregnancy and women were not just 'at risk' in the postnatal stage.

\section{Meaning of the study: possible mechanisms and implications for clinicians or policymakers}

Despite women reporting that they have a good QOL and are satisfied with their health, there is evidence of a significant burden of ill-health (including infectious, medical or obstetric, psychological and social morbidity) in women during pregnancy and up to 12 weeks after childbirth. Even though many women access care during pregnancy, at present the available care packages do not include comprehensive screening for all forms of 
ill-health. The focus till now has largely been on detection and prevention and treatment of HIV, malaria and syphilis and on emergency preparedness for birth. Although laboratory screening for anaemia is advised, this is rarely implemented. Screening for psychological or social ill-health rarely happens. Finally, treatment pathways for women who do have identified health needs are often not in place or of very poor quality.

\section{Unanswered questions and future research}

There is a need to refine and condense the current data collection tool, and to build expert consensus regarding the inclusion and weight given to each key indicator to develop a more concise but still representative and composite maternal morbidity assessment tool and score. Further research is required to assess the applicability of this score as a clinical outcome measure and a strategic and programmatic key performance indicator in different health systems both in a clinical and research capacity. There is also a need for qualitative research to enable a better understanding of what women, their families and their healthcare providers consider to be maternal morbidity, and, to understand the cultural context of how women report and describe ill-health including psychological morbidity and domestic violence. Anaemia and a positive SIRS screen as a marker of infection may be useful clinical proxy markers for physical maternal morbidity if more sophisticated diagnostic and clinical tests are not available or cannot be provided. This will require further study.

To be able to provide estimates of change in (any type of) maternal morbidity over the course of pregnancy, longitudinal cohort studies are needed with assessments of morbidity through the course of pregnancy and after childbirth. Similarly, additional data would need to be collected to assess associations between maternal morbidity, pregnancy outcomes and newborn health.

Acknowledgements A concept note to the Bill and Melinda Gates Foundation for a maternal morbidity programme was jointly developed by Professor Nynke van den Broek and Dr Lale Say. The maternal morbidity assessment tool used in this study was reviewed by members of the WHO Maternal Morbidity Working Group; Lale Say, Doris Chou, Priya Agrawal, Jose Guilherme Ceccatti, Peter von Dadelszen, Olubukola Fawole, Tabassum Firoz, Veronique Filippi, Atif Ghérissi, Gathari Ndirangu Gichuhi, Gill Gyte, Anoma Jayathilaka, Majorie Koblinsky, Yacouba Kone, Rachel van der Kruik, Isabelle Lange, Affette McCaw-Binns, Mark Morgan, Stephen Munjanja, Cihan Öztopcu, Elizabeth Sullivan, Özge Tunçalp. The authors would like to thank Dr Sunday Adaji, Katrin Metsis, Caroline Hercod and all research assistants and research supervisors in each healthcare facility for their participation.

Contributors MM co-ordinated and supervised the in-country data collection, collected data, conducted data analysis and wrote the manuscript. BM supervised and monitored all data collection in each country. SAW helped design the study, checked all data analysis and performed further analyses. FD formatted the tool for electronic data collection onto tablets and helped oversee the data collection. MA imported electronic data and cleaned, processed, coded the datasets and performed preliminary analysis. SB-Z supervised data collection in Malawi, PG in Kenya, PM in India and SZ in Pakistan. NvdB developed the study design and protocol, oversaw the design and conduct of the study, data collection and analysis and wrote the manuscript. All authors have read, edited and approved the final manuscript for submission.

Funding This study was funded by two grants to the Centre for Maternal and Newborn Health at the Liverpool School of Tropical Medicine in the UK: Global Health Grant (OPP1033805) from Bill and Melinda Gates Foundation via WHO and a grant from the Department of International Development, London, UK, under the Making it Happen programme (202945-101).

Disclaimer The funders played no role in the writing of the manuscript or the decision to submit it for publication.

Competing interests None declared.

Patient consent Obtained.

Ethics approval The Liverpool School of Tropical Medicine, Liverpool, UK, granted full ethical approval (LSTM14.025). Ethical approval was also obtained from each country-specific research ethics committee: The College of Medicine Research and Ethics Committee, College of Medicine, Blantyre, Malawi (P.07/14/1600); Kenyatta National Hospital and University of Nairobi, Ethics and Research Committee, Nairobi, Kenya (P574/09/214); Research and Ethics Committee, Vardhman Mahavir Medical College and Safdarjung Hospital, New Delhi, India (IEC/SJH/VMMC/Project/ September-14/19/482) and the National Bioethics Committee, Islamabad, Pakistan (4-87/14/NBC-159/RDC/1850).

Provenance and peer review Not commissioned; externally peer reviewed.

Open Access This is an Open Access article distributed in accordance with the Creative Commons Attribution Non Commercial (CC BY-NC 4.0) license, which permits others to distribute, remix, adapt, build upon this work non-commercially, and license their derivative works on different terms, provided the original work is properly cited and the use is non-commercial. See: http://creativecommons.org/ licenses/by-nc/4.0/

C Article author(s) (or their employer(s) unless otherwise stated in the text of the article) 2018. All rights reserved. No commercial use is permitted unless otherwise expressly granted.

\section{REFERENCES}

1. United Nations. Every Woman, Every Child: Global Strategy. New York: United Nations, 2015.

2. World Health Organization. Constitution of the World Health Organization as adapted by the International Health Conference. Geneva: World Health Organization, 1946.

3. United Nations. Transforming our world: the 2030 Agenda for Sustainable Development. New York: World Health Organization, 2015.

4. Liskin LS. Maternal morbidity in developing countries: a review and comments. Int J Gynaecol Obstet 1992;37:77-87.

5. Datta KK, Sharma RS, Razack PMA, et al. Morbidity pattern among rural women in Alwar-Rajasthan - A cohort study. Health Popul Perspect Issues 1980;3:282-92.

6. Koblinsky MA. Beyond maternal mortality--magnitude, interrelationship, and consequences of women's health, pregnancyrelated complications and nutritional status on pregnancy outcomes. Int J Gynaecol Obstet 1995;48:S21-S32.

7. Zafar S, Jean-Baptiste R, Rahman A, et al. Non-Life Threatening Maternal Morbidity: Cross Sectional Surveys from Malawi and Pakistan. PLoS One 2015;10:e0138026.

8. Say L, Souza JP, Pattinson RC. WHO working group on Maternal Mortality and Morbidity classifications. Maternal near miss-towards a standard tool for monitoring quality of maternal health care. Best Pract Res Clin Obstet Gynaecol 2009;23:287-96.

9. WHO, UNFPA, UNICEF. Monitoring emergency obstetric care a handbook. Geneva, Switzerland: World Health Organization, 2009.

10. Vos T, Barber RM, Bell B, et al. Global, regional, and national incidence, prevalence, and years lived with disability for 301 acute and chronic diseases and injuries in 188 countries, 1990-2013: a systematic analysis for the Global Burden of Disease Study 2013. Lancet 2015;386:743-800.

11 Vanderkruik RC, Tunçalp Ö, Chou D, et al. Framing maternal morbidity: WHO scoping exercise. BMC Pregnancy Childbirth 2013;13:213.

12. Ugandan Maternal Child/Family Planning Division. Evaluation of maternal morbidity and mortality in 12 selected districts of Uganda (March 1993). Entebbe, Uganda: Ministry of Health, Maternal Child/ Family Planning Division, 1994.

13. World Health Organization. World Health Statistics 2015. Geneva: World Health Organization, 2015.

14. World Health Organization. World Health Statistics 2017: Monitoring health for SDGs. Geneva: World Health Organization, 2017.

15. Kuppuswamy B. Manual of Socioeconomic Status (urban). Delhi: Manasayan, 1981. 
16. Jolliffe I. Principal Component Analysis. London: John Wiley \& Sons, Ltd, 2014.

17. Filmer D, Pritchett LH. Estimating wealth effects without expenditure data--or tears: an application to educational enrollments in states of India. Demography 2001;38:115-32.

18. Cox JL, Holden JM, Sagovsky R. Detection of postnatal depression. Development of the 10-item Edinburgh Postnatal Depression Scale. Br J Psychiatry 1987;150:782-6.

19. World Health Organization. WHOQOL-SRPB Field-Test Instrument Geneva: Department of Mental Health \& Substance Dependence, World Health Organization, 2002.

20. Sherin KM, Sinacore JM, Li XQ, et al. HITS: a short domestic violence screening tool for use in a family practice setting. Fam Med 1998;30:508-12.

21. World Health Organization. Alcohol, Smoking and Substance Involvement Screening Test (ASSIST). Geneva: World Health Organization, 2010.

22. World Health Organization. Haemoglobin concentrations for the diagnosis of anaemia and assessment of severity. Geneva: World Health Organization, 2011.

23. Brown MA, Lindheimer MD, de Swiet M, et al. The classification and diagnosis of the hypertensive disorders of pregnancy: statement from the International Society for the Study of Hypertension in Pregnancy (ISSHP). Hypertens Pregnancy 2001;20:ix-xiv.
24. GBD 2015 DALYs and HALE Collaborators. Global, regional, and national disability-adjusted life-years (DALYs) for 315 diseases and injuries and healthy life expectancy (HALE), 1990-2015: a systematic analysis for Global Burden of Disease Study 2015. Lancet 2015(388):1603-58.

25. Osman-Hassan E. Study of the prevalence and perception of maternal morbidity in Menoufeya Governate, Egypt. Cairo: The Egyptian Fertility Care Society, 1995.

26. Bhatia JC. Levels and determinants of maternal morbidity: results from a community-based study in southern India. Int $J$ Gynaecol Obstet 1995;50(Suppl 2):S153-63.

27. Gibson J, McKenzie-McHarg K, Shakespeare J, et al. A systematic review of studies validating the Edinburgh Postnatal Depression Scale in antepartum and postpartum women. Acta Psychiatr Scand 2009;119:350-64.

28. Sawyer A, Ayers S, Smith H. Pre- and postnatal psychological wellbeing in Africa: a systematic review. J Affect Disord 2010;123:17-29.

29. Fisher J, Cabral de Mello M, Patel V, et al. Prevalence and determinants of common perinatal mental disorders in women in low- and lower-middle-income countries: a systematic review. Bull World Health Organ 2012;90:139-49.

30. Gelaye B, Rondon MB, Araya R, et al. Epidemiology of maternal depression, risk factors, and child outcomes in low-income and middle-income countries. Lancet Psychiatry 2016;3:973-82. 